\title{
THERMOELASTIC PROBLEM OF AN AXIALLY MOVING MICROBEAM SUBJECTED TO AN EXTERNAL TRANSVERSE EXCITATION
}

\author{
Ahmed E. Abouelregal \\ Department of Mathematics, Faculty of Science, Mansoura University, Mansoura, Egypt \\ Department of Mathematics, College of Science and Arts, University of Aljouf, El-Qurayat, Saudi Arabia
}

\author{
Ashraf M. Zenkour \\ Department of Mathematics, Faculty of Science, King Abdulaziz University, Jeddah, Saudi Arabia \\ Department of Mathematics, Faculty of Science, Kafrelsheikh University, Kafr El-Sheikh, Egypt \\ e-mail: zenkour@kau.edu.sa
}

\begin{abstract}
In this study, the problem of an axially moving microbeam subjected to sinusoidal pulse heating and an external transverse excitation is solved. The generalized thermoelasticity theory with one relaxation time is used to solve this problem. An analytical technique based on the Laplace transform is used to calculate the vibration of deflection and the temperature. The inverse of Laplace transforms are computed numerically using Fourier expansion techniques. The effects of the pulse-width of thermal vibration, moving speed and the transverse excitation are studied and discussed on the lateral vibration, temperature, displacement and bending moment of the beam.
\end{abstract}

Keywords: thermoelasticity, microbeam, sinusoidal pulse heating, axially moving, transverse excitation

\section{Introduction}

The uncoupled thermoelasticity theory predicts two phenomena not compatible with physical observations. First, the equation of heat conduction of this theory does not contain any elastic terms contrary to the fact that elastic changes produce heat effects. Second, the heat equation is of parabolic type predicting infinite speed of propagation for heat waves. Biot (1956) formulated the theory of coupled thermoelasticity to overcome the first shortcoming. The governing equations of this theory are coupled, eliminating the first paradox in the classical uncoupled theory. However, both theories share the second shortcoming since the heat equation for the coupled theory implies also infinite speed of heat waves.

Lord and Shulman (1967) introduced the theory of generalized thermoelasticity with one relaxation time for the special case of an isotropic body. In this theory, a modified law of heat conduction including both the heat flux and its time derivative replaces the conventional Fourier's law. The heat equation associated with this theory is hyperbolic and hence eliminates the paradox of infinite speeds of propagation inherent in both the uncoupled and the coupled theories of thermoelasticity. Green and Lindsay (1972) obtained the theory of thermoelasticity with two relaxation times. In this theory, the classical Fourier's law of heat conduction is not violated when the body under consideration has a center of symmetry.

The investigations on transverse vibrations of axially moving strings have theoretical significance as well. Many engineering devices, such as power transmission bands, belt saws, aerial cable tramways, elevator cables, can be modeled as axially moving beams if the bending stiffness needs to be considered. Under certain excitations, axially moving beams undergo the transverse motion. If the motion amplitude is large, the nonlinearity needs to be taken into account. Therefore, understanding nonlinear vibrations of axially moving beams is significant for the design 
of devices. The governing equations of axially moving beams, containing a term of the mixed partial derivative with respect to time and space, are a typical gyroscopic continuum. The related investigations also have theoretical significance. Most of the investigations concern the transverse vibration characteristics and dynamical behavior of an axially moving beam.

Study of axially moving beams or strings originated from the work by Mote (1965) where the natural frequencies of axially moving strings were found to decrease with translating speed. Beams with lengths that change over time were studied by Tabarrok and Leech (1965). Chakraborty and Malik (2000) analyzed linear and nonlinear free vibration of the axially moving simply supported beam by wave propagation. Kong and Parker (2004) researched free vibration of the axially moving beam with small flexural stiffness. Asymptotic solutions of natural frequencies were obtained by the perturbation method of algebraic equation. Yang (2007) studied natural frequencies of axially moving beams by the method of multiple scales.

The above mentioned researches are all in a stable temperature field, the impacts of temperature changes are ignored. But in the actual engineering systems, the impacts of temperature changes need to be taken into consideration. The bending of the beam is inevitably affected by the temperature changes in elastic vibration, vibration characteristics and dynamics of the beam will be changed due to the variation of temperature (Avsec and Oblak, 2007; Manoach and Ribeiro, 2004). For this reason, the research of coupled thermoelastic vibration characteristics for engineering system components has become a new research field. Owing to the temperature and displacement coupled field, the equation of thermal conduction involving the deformation term and the equation of motion must be coupled solved. Chang and Wan (1986) studied the nonlinear coupled thermoelastic vibration of an isotropic rectangular thin plate under all kinds of boundary conditions by the Berger assumption. Houston et al. (2004) studied the importance of thermoelastic damping for silicon-based MEMS.

In the present paper, the solution for generalized thermoelastic vibration of a microbeam resonator induced by sinusoidal pulse heating based on the Euler-Bernoulli beam theory is developed. The Laplace transform method is used to determine the lateral vibration, temperature, displacement, stress and strain of the beam. The effects of speed, excitation, and width of the sinusoidal pulse parameters are studied and represented graphically.

\section{Basic equations}

Let us consider a homogenous isotropic thermoelastic solid in the Cartesian coordinate system $O x y z$, initially un-deformed and at uniform temperature $T_{0}$. The basic governing field equations upon employing linear generalized thermoelasticity for a solid in the absence of body forces, equilibrated forces and heat sources for the displacement vector $\mathbf{u}(x, y, z, t)$ are given by Lord and Shulman (1967)

$$
(\lambda+\mu) \nabla(\nabla \cdot \mathbf{u})+\mu \nabla^{2} \mathbf{u}-\gamma \nabla \theta=\rho \frac{\partial^{2} \mathbf{u}}{\partial t^{2}}
$$

The heat conduction equation corresponding to the generalized theory of thermoelasticity proposed by to Lord-Shulman takes the form

$$
K \nabla^{2} \theta+\left(1+\tau_{0} \frac{\partial}{\partial t}\right)(\rho Q)=\left(1+\tau_{0} \frac{\partial}{\partial t}\right)\left(\rho C_{E} \frac{\partial \theta}{\partial t}+\gamma T_{0} \frac{\partial e}{\partial t}\right)
$$

The constitutive relations considering the effect of temperature have the form

$$
\sigma_{i j}=2 \mu \varepsilon_{i j}+\lambda u_{r, r} \delta_{i j}-\gamma \theta \delta_{i j}
$$


where $\sigma_{i j}$ are components of the stress tensor and $\varepsilon_{i j}$ are components of the strain tensor given by

$$
\varepsilon_{i j}=\frac{1}{2}\left(u_{i, j}+u_{j, i}\right)
$$

where $K$ is the thermal conductivity, $C_{E}$ is the specific heat per unit mass at constant strain, $\theta=T-T_{0}$ is the temperature increment of the resonator, in which $T_{0}$ is the environmental temperature and $T(x, z, t)$ is the temperature distribution, $\gamma=E \alpha_{T} /(1-2 \nu)$ in which $\alpha_{T}$ is the thermal expansion coefficient, $e=u_{r, r}$ is the cubical dilatation, $E$ is Young's modulus, $\nu$ is Poisson's ratio, $\rho$ is the material density of the medium, $\tau_{0}$ is the relaxation time, and $Q$ is the heat source. The thermal relaxation time $\tau_{0}$ can be physically interpreted as the "time-lag needed to establish the steady state of heat conduction in a volume element when a temperature gradient is suddenly imposed on that element". When $\tau_{0}$ vanish identically, the LS theory reduces to the classical thermoelasticity theory.

\section{Formulation of the problem}

We consider a homogeneous isotropic thermoelasitc axially moving beam with the cross-sectional area $A$ and moment of inertia $I$, which travels at a constant axial speed $v$ between two motionless ends separated by the distance $L$ as shown in Fig. 1 . The beam is subjected to an external transverse excitation $F(x, t)$, where $t$ is time and $x$ is the axial coordinate. The beam has length $L$, width $b$ and thickness $h$ in the $x, y$ and $z$ directions, respectively.

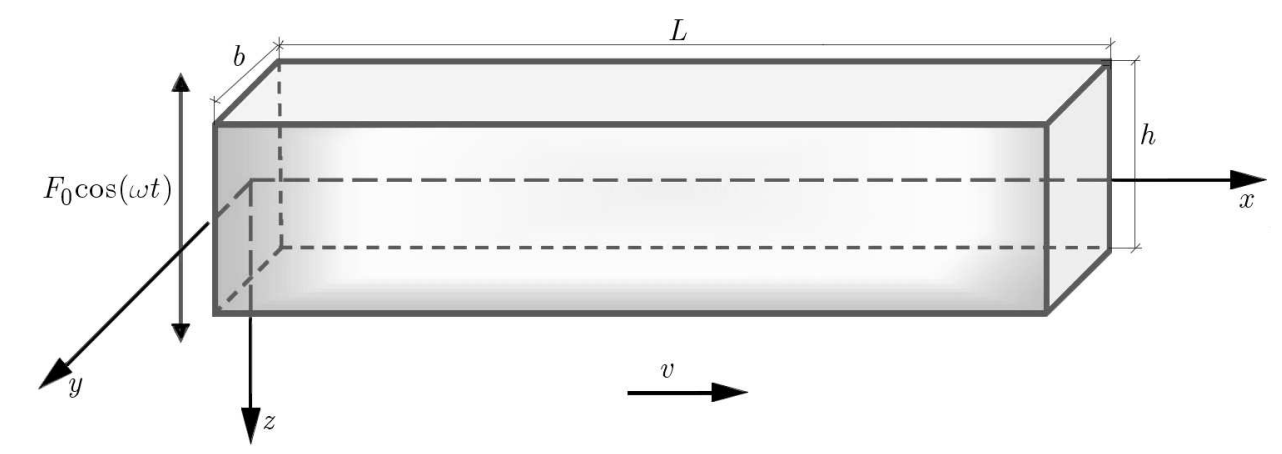

Fig. 1. A microbeam axially moving with a constant speed $v$ and subjected to an external excitation

The beam undergoes bending vibrations of small amplitude about the $x$-axis such that the deflection is consistent with the linear Euler-Bernoulli theory. That is, any plane cross-section initially perpendicular to the axis of the beam remains plane and perpendicular to the neutral surface during bending. Only the bending vibration described by the transverse displacement $w(x, t)$ (lateral deflection) is considered. Thus, the displacements are given by

$$
u_{1}=u=-z \frac{\partial w}{\partial x} \quad u_{2}=0 \quad u_{3}(x, y, z, t)=w(x, t)
$$

The Newton second law of motion yields (Ding and Chen, 2011; Chen and Yang, 2005)

$$
\frac{\partial^{2} M}{\partial x^{2}}+\rho A\left(\frac{\partial^{2} w}{\partial t^{2}}+2 v \frac{\partial^{2} w}{\partial x \partial t}+v^{2} \frac{\partial^{2} w}{\partial x^{2}}\right)=F(x, t)
$$

where $M(x, t)$ is the bending moment.

The one-dimensional constitutive equation can be written as

$$
\sigma_{x}=-E\left(z \frac{\partial^{2} w}{\partial x^{2}}+\alpha_{T} \theta\right)
$$

where $\sigma_{x}$ is the axial stress. 
According to the linear beam theory of Euler-Bernoulli, the bending moment of the cross-section is given by

$$
M(x, t)=-b \int_{-h / 2}^{h / 2} \sigma_{x} z d z
$$

Multiplying both sides of Eq. (3.3) by $z$ and integrating over the cross-section area of the beam, we find that the flexural moment of the cross-section stisfies the equation

$$
M(x, t)=E I\left(\frac{\partial^{2} w}{\partial x^{2}}+\alpha_{T} M_{T}\right)
$$

where $I=b h^{3} / 12$ is the inertia moment of the cross-section, $E I$ is the flexural rigidity of the beam and $M_{T}$ is the thermal moment

$$
M_{T}=\frac{12}{h^{3}} \int_{-h / 2}^{h / 2} \theta(x, z, t) z d z
$$

Substituting Eq. (3.5) into Eq. (3.2), one can get the equation of motion of the beam as

$$
\frac{\partial^{4} w}{\partial x^{4}}+\frac{\rho A}{E I}\left(\frac{\partial^{2} w}{\partial t^{2}}+2 v \frac{\partial^{2} w}{\partial x \partial t}+v^{2} \frac{\partial^{2} w}{\partial x^{2}}\right)-\frac{1}{E I} F(x, t)+\alpha_{T} \frac{\partial^{2} M_{T}}{\partial x^{2}}=0
$$

Substituting the Euler-Bernoulli assumption into Eq. (2.2) gives the thermal conduction equation for the beam without the heat source $(Q=0)$, as

$$
K\left(\frac{\partial^{2} \theta}{\partial x^{2}}+\frac{\partial^{2} \theta}{\partial z^{2}}\right)=\left(1+\tau_{0} \frac{\partial}{\partial t}\right)\left[\rho C_{E} \frac{\partial \theta}{\partial t}-\gamma T_{0} z \frac{\partial}{\partial t}\left(\frac{\partial^{2} w}{\partial x^{2}}\right)\right]
$$

\section{Solution of the problem}

For a very thin beam (microbeam), assuming that the temperature increment varies in terms of a $\sin (p z)$ function (sinusoidal variation) along the thickness direction, where $p=\pi / h$

$$
\theta(x, z, t)=\Theta(x, t) \sin (p z)
$$

Thus, Eqs. (3.5) and (3.7) become

$$
\begin{aligned}
& M(x, t)=E I\left(\frac{\partial^{2} w}{\partial x^{2}}+\frac{24 \alpha_{T}}{h \pi^{2}} \Theta(x, t)\right) \\
& \frac{\partial^{4} w}{\partial x^{4}}+\frac{\rho A}{E I}\left(\frac{\partial^{2} w}{\partial t^{2}}+2 v \frac{\partial^{2} w}{\partial x \partial t}+v^{2} \frac{\partial^{2} w}{\partial x^{2}}\right)-\frac{1}{E I} F(x, t)+\frac{24 \alpha_{T}}{h \pi^{2}} \frac{\partial^{2} \Theta}{\partial x^{2}}=0
\end{aligned}
$$

Now, multiplying Eq. (3.8) by $12 z / h^{3}$ and integrating it with respect to $z$ through the beam thickness from $-h / 2$ to $h / 2$, yields

$$
\left[\left(\frac{\partial^{2}}{\partial x^{2}}-\frac{\pi^{2}}{h^{2}}\right)-\eta\left(1+\tau_{0} \frac{\partial}{\partial t}\right) \frac{\partial}{\partial t}\right] \Theta=-\frac{\gamma T_{0} \pi^{2} h}{24 K}\left(1+\tau_{0} \frac{\partial}{\partial t}\right) \frac{\partial^{3} w}{\partial t \partial x^{2}}
$$

where $\eta=\rho C_{E} / K$. 
Now, for simplicity, we will use the following dimensionless variables

$$
\begin{array}{lll}
\left(x^{\prime}, L^{\prime}, u^{\prime}, w^{\prime}, z^{\prime}, h^{\prime}, b^{\prime}\right)=\eta c(x, L, u, w, z, h, b) & \left(t^{\prime}, t_{0}^{\prime}\right)=\eta c^{2}\left(t, t_{0}\right) \\
\Theta^{\prime}=\frac{\Theta}{T_{0}} & \sigma_{x}^{\prime}=\frac{\sigma_{x}}{E} & M^{\prime}=\frac{M}{\eta c E I} \\
F^{\prime}=\frac{A F}{E I} & v^{\prime}=\frac{v}{c} & c=\sqrt{\frac{E}{\rho}}
\end{array}
$$

So, the governing equations and the bending and constitutive equations in nondimensional forms are simplified as (dropping the primes for convenience)

$$
\begin{aligned}
& \frac{\partial^{4} w}{\partial x^{4}}+A_{1}\left(\frac{\partial^{2} w}{\partial t^{2}}+2 v \frac{\partial^{2} w}{\partial x \partial t}+v^{2} \frac{\partial^{2} w}{\partial x^{2}}-F\right)+A_{2} \frac{\partial^{2} \Theta}{\partial x^{2}}=0 \\
& {\left[\frac{\partial^{2}}{\partial x^{2}}-\left(1+\tau_{0} \frac{\partial}{\partial t}\right) \frac{\partial}{\partial t}-A_{4}\right] \Theta=-A_{5}\left(1+\tau_{0} \frac{\partial}{\partial t}\right) \frac{\partial^{3} w}{\partial t \partial x^{2}}} \\
& M(x, t)=\frac{\partial^{2} w}{\partial x^{2}}+A_{2} \Theta(x, t) \quad \sigma_{x}=-z \frac{\partial^{2} w}{\partial x^{2}}-A_{3} \Theta(x, t) \sin (p z)
\end{aligned}
$$

where

$$
A_{1}=\frac{12}{h^{2}} \quad A_{2}=\frac{24 T_{0} \alpha_{T}}{\pi^{2} h} \quad A_{3}=\alpha_{T} T_{0} \quad A_{4}=\frac{\pi^{2}}{h^{2}} \quad A_{5}=\frac{\gamma \pi^{2} h}{24 K \eta}
$$

A special form of the external transverse excitation is considered. It is assumed that the excitation is spatially uniform and temporally harmonic, i.e.

$$
F(x, t)=F_{0} \cos (\omega t)
$$

where $F_{0}$ and $\omega$ are the dimensionless amplitude and frequency of the external excitation, respectively.

\section{Initial and boundary conditions}

In order to solve the problem, both initial and boundary conditions need to be considered. The initial conditions of the problem are assumed to be homogeneous. The initial conditions are taken as

$$
\left.w(x, t)\right|_{t=0}=\left.\frac{\partial w(x, t)}{\partial t}\right|_{t=0}=\left.0 \quad \Theta(x, t)\right|_{t=0}=\left.\frac{\partial \Theta(x, t)}{\partial t}\right|_{t=0}=0
$$

These initial conditions are supplemented by considering that the two ends of the microbeam are clamped (Zenkour et al., 2014)

$$
\left.w(x, t)\right|_{x=0, L}=\left.0 \quad \frac{\partial w(x, t)}{\partial x}\right|_{x=0, L}=0
$$

Let us also consider that the microbeam is thermally loaded by sinusoidal pulse heating incidents into the surface of the microbeam $x=0$ with pulse width $t_{0}$. In this case, we take the conductive temperature in the form (Zenkour and Abouelregal, 2014)

$$
\left.\Theta(x, t)\right|_{x=0}=f(t)= \begin{cases}\sin \frac{\pi t}{t_{0}} & 0 \leqslant t \leqslant t_{0} \\ 0 & t>t_{0}, \quad t<0\end{cases}
$$

In addition, the temperature at the end boundary should satisfy the following relation

$$
\frac{\partial \Theta}{\partial x}=0 \quad x=L
$$




\section{Solution in the Laplace transform domain}

Applying the Laplace transform defined by the relation

$$
\bar{g}(x, s)=\int_{0}^{\infty} g(x, s) e^{-s t} d t
$$

to both sides of Eqs. $(4.5)_{1,2}$, gives

$$
\begin{aligned}
& \frac{d^{4} \bar{w}}{d x^{4}}+v^{2} A_{1} \frac{d^{2} \bar{w}}{d x^{2}}+2 v s A_{1} \frac{d \bar{w}}{d x}+A_{1} s^{2} \bar{w}-\bar{F}=-A_{2} \frac{d^{2} \bar{\Theta}}{d x^{2}} \\
& \frac{d^{2} \bar{\Theta}}{d x^{2}}-A_{6} \bar{\Theta}=-A_{7} \frac{d^{2} \bar{w}}{d x^{2}}
\end{aligned}
$$

where

$$
A_{6}=\frac{s\left(1+\tau_{q} s\right)}{1+\tau_{\theta} s}+A_{4} \quad A_{7}=\frac{s A_{5}\left(1+\tau_{q} s\right)}{1+\tau_{\theta} s} \quad \bar{F}=\frac{s}{s^{2}+\omega^{2}}
$$

Note that the overbar symbol denotes its Laplace transform and $s$ denotes the Laplace transform parameter. Elimination $\bar{\Theta}$ or $\bar{w}$ from Eqs. (6.2) gives the following differential equations for either $\bar{w}$ or $\bar{\Theta}$

$$
\begin{aligned}
& \left(\frac{d^{6}}{d x^{6}}+a_{4} \frac{d^{4}}{d x^{4}}+a_{3} \frac{d^{3}}{d x^{2}}+a_{2} \frac{d^{2}}{d x^{2}}+a_{1} \frac{d}{d x}+a_{0}\right) \bar{w}=-A_{6} \bar{F} \\
& \left(\frac{d^{6}}{d x^{6}}+a_{4} \frac{d^{4}}{d x^{4}}+a_{3} \frac{d^{3}}{d x^{2}}+a_{2} \frac{d^{2}}{d x^{2}}+a_{1} \frac{d}{d x}+a_{0}\right) \bar{\Theta}=0
\end{aligned}
$$

where the coefficients $a_{i}, i=0, \ldots, 4$ are given by

$$
\begin{aligned}
& a_{4}=-A_{6}+A_{1} v^{2}-A_{2} A_{7} \quad a_{3}=2 s v A_{1} \quad a_{2}=A_{1} s^{2}-A_{6} A_{1} v^{2} \\
& a_{1}=-2 s A_{1} A_{6} v \quad a_{0}=-s^{2} A_{1} A_{6}
\end{aligned}
$$

The solution to governing equations, Eqs. $(6.2)_{2}$, in the Laplace transformation domain can be represented as

$$
\bar{w}=\sum_{i=1}^{6} C_{i} e^{m_{i} x}-\frac{A_{6} \bar{F}}{a_{0}} \quad \bar{\Theta}=\sum_{i=1}^{6} C_{i}^{\prime} e^{m_{i} x}
$$

where $C_{i}$ and $C_{i}^{\prime}$ are parameters depending on $s$ and and $m_{i}, i=1,2, \ldots, 6$ are the roots of the characteristic equation

$$
m^{6}+a_{4} m^{4}+a_{3} m^{3}+a_{2} m^{2}+a_{2} m+a_{0}=0
$$

Kulkarni (2008) described a method to decompose the given six-degree polynomial equation into two cubic polynomials as factors. The cubic polynomials are then equated to zero and solved to obtain the six roots of Eq. (6.7) in radicals. The salient feature of the equation solved in this manner is that the sum of its three roots is equal to the sum of its remaining three roots. He derived the condition required to be satisfied by the coefficients of such a solvable equation.

Consider another six-degree polynomial equation as shown below

$$
\left(m^{3}+b_{2} m^{2}+b_{1} m+b_{0}\right)^{2}-\left(c_{2} m^{2}+c_{1} m+c_{0}\right)^{2}=0
$$

where $b_{0}, b_{1}, b_{2}$ and $c_{0}, c_{1}, c_{2}$ are unknown coefficients in the constituent cubic and quadratic polynomials respectively, in the above equation. Therefore, if the given six-degree equation, Eq. 
(6.7), can be represented in form of Eq. (6.8), then it can be factored into two cubic polynomial expresions, leading to its solution. These polynomial expresions are equated to zero to obtain the following cubic equations

$$
\begin{aligned}
& m^{3}+\left(b_{2}-c_{2}\right) m^{2}+\left(b_{1}-c_{1}\right) m+b_{0}-c_{0}=0 \\
& m^{3}+\left(b_{2}+c_{2}\right) m^{2}+\left(b_{1}+c_{1}\right) m+b_{0}+c_{0}=0
\end{aligned}
$$

The six roots of the given six-degree equation are then obtained by solving the above cubic equations. Thus in order to represent Eq. (6.7) in form of Eq. (6.8), the coefficients of the two equations should be equal. This gives

$$
\begin{array}{lll}
b_{0}=\frac{a_{3}}{2} & b_{1}=\frac{a_{4}}{2} & b_{2}=0 \\
c_{2}=0 & c_{1}^{2}=\frac{a_{4}^{4}}{4}-a_{2}= \pm a_{5} & c_{0}^{2}=\frac{a_{3} a_{4}}{4}-\frac{a_{1}}{2}= \pm a_{6}
\end{array}
$$

We have determined all the unknowns, and substituting their values in cubic Eqs. (6.9), we get the following pair of cubic equations

$$
\begin{aligned}
& m^{3}+\left(b_{1}-a_{6}\right) m+b_{0}-a_{7}=0 \\
& m^{3}+\left(b_{1}+a_{6}\right) m+b_{0}+a_{7}=0
\end{aligned}
$$

where $a_{7}=a_{6} / a_{5}$. These equations are then solved to obtain all the six roots of Eq. (6.7) in the form

$$
\begin{aligned}
m_{1} & =\frac{2}{3} p_{1} \sin q_{1} & m_{2} & =-\frac{1}{3} p_{1}\left(\sqrt{3} \cos q_{1}+\sin q_{1}\right) \\
m_{3} & =\frac{1}{3} p_{1}\left(\sqrt{3} \cos q_{1}-\sin q_{1}\right) & m_{4} & \left.=\frac{2}{3} p_{2} \sin q_{2}\right) \\
m_{5} & =-\frac{1}{3} p_{2}\left(\sqrt{3} \cos q_{2}+\sin q_{2}\right) & m_{6} & =\frac{1}{3} p_{2}\left(\sqrt{3} \cos q_{2}-\sin q_{2}\right)
\end{aligned}
$$

where

$$
\begin{array}{ll}
p_{1}=\sqrt{3\left(a_{6}-b_{1}\right)} & q_{1}=\frac{1}{3} \sin ^{-1} \frac{27\left(b_{0}-a_{7}\right)}{2 p_{1}^{3}} \\
p_{2}=\sqrt{3}\left(a_{6}+b_{1}\right) & q_{2}=\frac{1}{3} \sin ^{-1} \frac{27\left(b_{0}+a_{7}\right)}{2 p_{2}^{3}}
\end{array}
$$

The compatibility between Eqs. (6.6) and Eq. (6.2) 2 , gives

$$
C_{i}^{\prime}=\beta_{i} C_{i} \quad \beta_{i}=-\frac{A_{7} m_{i}^{2}}{m_{i}^{2}-A_{6}}
$$

Then

$$
\bar{\Theta}=\sum_{i=1}^{6} \beta_{i} C_{i} e^{m_{i} x}
$$

Then, the thermodynamical temperature $\bar{\theta}$ and the axial thermal stress $\bar{\sigma}_{x}$ given in Eqs. (4.1) and $(4.5)_{4}$ in the Laplace domain with the aid of Eqs. (6.6) $)_{1}$, become

$$
\bar{\theta}=\sin \left(\frac{\pi z}{h}\right) \sum_{i=1}^{6} \beta_{i} C_{i} e^{m_{i} x} \quad \bar{\sigma}_{x}=-\sum_{i=1}^{6}\left[z m_{i}^{2}+A_{3} \sin \left(\frac{\pi z}{h}\right) \beta_{i}\right] C_{i} e^{m_{i} x}
$$


Also, the axial displacement after using Eq. (6.6) 1 takes the form

$$
\bar{u}=-z \frac{d \bar{w}}{d x}=-z \sum_{i=1}^{6} m_{i} C_{i} e^{m_{i} x}
$$

In addition, the dilatation strain will be

$$
\bar{e}=\frac{d \bar{u}}{d x}=-z \sum_{i=1}^{6} m_{i}^{2} C_{i} e^{m_{i} x}
$$

Using the Laplace transformation to Eqs. (5.2)-(5.4), the boundary conditions take the forms

$$
\begin{array}{ll}
\left.\bar{w}(x, s)\right|_{x=0, L}=0 & \left.\frac{d \bar{w}(x, s)}{d x}\right|_{x=0, L}=0 \\
\left.\bar{\Theta}(x, s)\right|_{x=0}=\frac{\pi t_{0}}{\pi^{2}+t_{0}^{2} s^{2}}=\bar{G}(s) & \left.\frac{d \bar{\Theta}(x, s)}{d x}\right|_{x=L}=0
\end{array}
$$

Substituting Eqs. (6.4) into the above boundary conditions, one obtains six linear equations in the matrix form as

$$
\left[\begin{array}{cccccc}
1 & 1 & 1 & 1 & 1 & 1 \\
e^{m_{1} L} & e^{m_{2} L} & e^{m_{3} L} & e^{m_{4} L} & e^{m_{5} L} & e^{m_{6} L} \\
m_{1} & m_{2} & m_{3} & m_{4} & m_{5} & m_{6} \\
m_{1} e^{m_{1} L} & m_{2} e^{m_{2} L} & m_{3} e^{m_{3} L} & m_{4} e^{m_{4} L} & m_{5} e^{m_{5} L} & m_{6} e^{m_{6} L} \\
\beta_{1} & \beta_{2} & \beta_{3} & \beta_{4} & \beta_{5} & \beta_{6} \\
\beta_{1} m_{1} e^{m_{1} L} & \beta_{2} m_{2} e^{m_{2} L} & \beta_{3} m_{3} e^{m_{3} L} & \beta_{4} m_{4} e^{m_{4} L} & \beta_{5} m_{5} e^{m_{5} L} & \beta_{6} m_{6} e^{m_{6} L}
\end{array}\right]\left[\begin{array}{c}
C_{1} \\
C_{2} \\
C_{3} \\
C_{4} \\
C_{5} \\
C_{6}
\end{array}\right]=\left[\begin{array}{c}
A_{6} \bar{F} \\
a_{0} \\
0 \\
0 \\
0 \\
\bar{G}(s) \\
0
\end{array}\right]
$$

The solution of the above system of linear equations gives the unknown parameters $C_{i}$ and $C_{i+3}$. This completes the solution to the problem in the Laplace transform domain.

\section{Inversion of the Laplace transforms}

In order to determine the conductive and thermal temperature, displacement and stress distributions in the time domain, the Riemann-sum approximation method is used to obtain the numerical results. In this method, any function in the Laplace domain can be inverted to the time domain as

$$
f(t)=\frac{e^{\zeta t}}{t}\left[\frac{1}{2} \Re[\bar{F}(\zeta)]+\Re \sum_{n=0}^{N} \bar{F}\left(\zeta+\frac{\mathrm{i} n \pi}{t}\right)(-1)^{n}\right]
$$

where $\Re$ is the real part and $\mathrm{i}$ is the imaginary number. For faster convergence, numerical experiments have shown that the value that satisfies the above relation is $\zeta=4.7 / t$ (Tzou 1996).

\section{Numerical results}

In terms of the Riemann-sum approximation defined in Eq. (7.1), numerical Laplace inversion is performed to obtain the non-dimensional temperature, displacement and stress in the beam. 
In the present work, the thermoelastic coupling effect is analyzed by considering a beam made of silicon. The material parameters are given as

$$
\begin{array}{llll}
E=169 \mathrm{GPa} & \rho=2330 \frac{\mathrm{kg}}{\mathrm{m}^{3}} & C_{E}=713 \frac{\mathrm{J}}{\mathrm{kgK}} & T_{0}=293 \mathrm{~K} \\
\frac{b}{h}=0.5 & \alpha_{T}=2.59 \cdot 10^{-6} \mathrm{~K}^{-1} & \nu=0.22 & K=156 \frac{\mathrm{W}}{\mathrm{mK}} \\
\frac{L}{h}=10 & t=0.12 \mathrm{~s} & &
\end{array}
$$

Equation (7.1) is used to invert the Laplace transforms in Eqs. (6.16)-(6.18) to graphically present the lateral vibration $w$, temperature $\theta$, axial displacement $u$, and the bending moment $M$ distributions with respect to the $x$ direction $(z=h / 6)$. The dimensionless transport speed $\bar{v}$ is considered such that $\bar{v}=10^{-3} v$. Numerical calculations are carried out for three cases.

The first case is to investigate the dimensionless lateral vibration, temperature, displacement, and moment with different transport speeds when the pulse-width and the frequency of the external excitation remain constant $\left(t_{0}=0.1, \omega=5\right)$. The effects of $\bar{v}$ on the vibration characteristics along the axial direction of the microbeam are shown in Fig. 2. The field quantities are very sensitive to the variation of the transport speed $\bar{v}$.
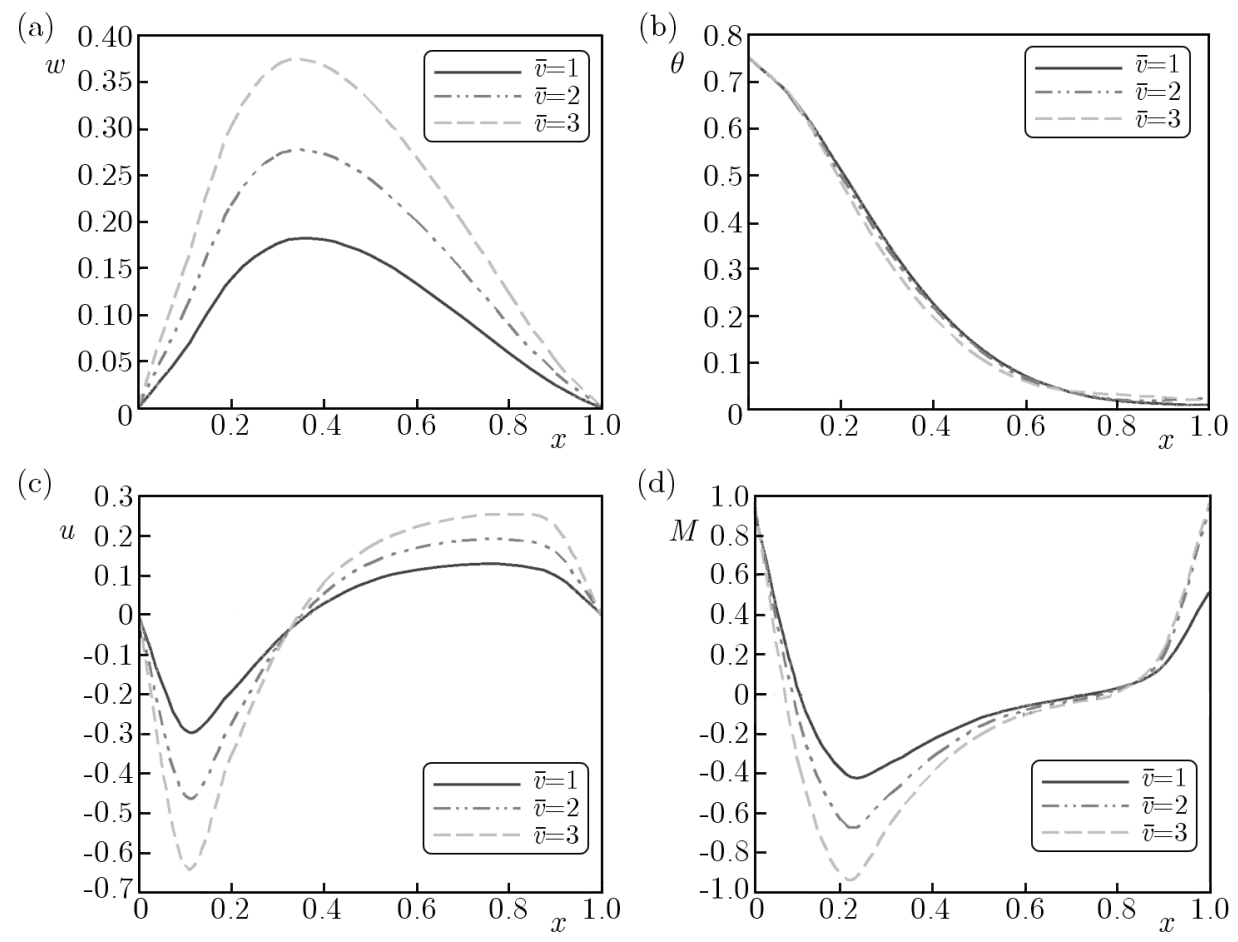

Fig. 2. Effect of the speed $\bar{v}$ on the field quantities along the axial direction of the moving microbeam;

(a) $w$ versus $x$, (b) $\theta$ versus $x$, (c) $u$ versus $x$, (d) $M$ versus $x$

It is observed in Fig. 2a that the distribution of lateral vibration $w$ vanishes at the ends of the microbeam to satisfy the boundary condition. The lateral vibration $w$ is no longer increasing and has its maximum depending on the value of the transport speed $\bar{v}$. The distribution of the waves of $w$ is increasing with an increase in the transport speed $\bar{v}$. Figure $2 \mathrm{~b}$ shows that the temperature $\theta$ decreases directly as the distance $x$ increases to move in the direction of wave propagation. The effect of the transport speed $\bar{v}$ is little pronounced. Figures $2 \mathrm{c}$ and $2 \mathrm{~d}$ show that the displacement and bending moment move directly in the direction of wave propagation and have different behavior. From these figures, we note that the presence of transport speed $\bar{v}$ has large effect on the displacement and bending moment. 
The second case is the investigation of the dimensionless field quantities with variation of the pulse-width parameter $t_{0}$ for $\bar{v}=1$ and $\omega=5$. The numerical results are obtained and presented graphically in Fig. 3. A significant effect of the pulse-width parameter $t_{0}$ on all the studied fields is seen there. An increase in the value of the pulse-width parameter causes a decrease in the lateral vibration and temperature fields, which it is very obvious in the peek points of the curves. Also, the values of the displacement $u$ start increasing with the pulse-width parameter in the range $0 \leqslant x \leqslant 0.38$, thereafter it increases to its maximum amplitudes in the range $0.38 \leqslant x \leqslant 1$. It can be observed that the pulse width parameter $t_{0}$ has a great effect on the distribution of field quantities.
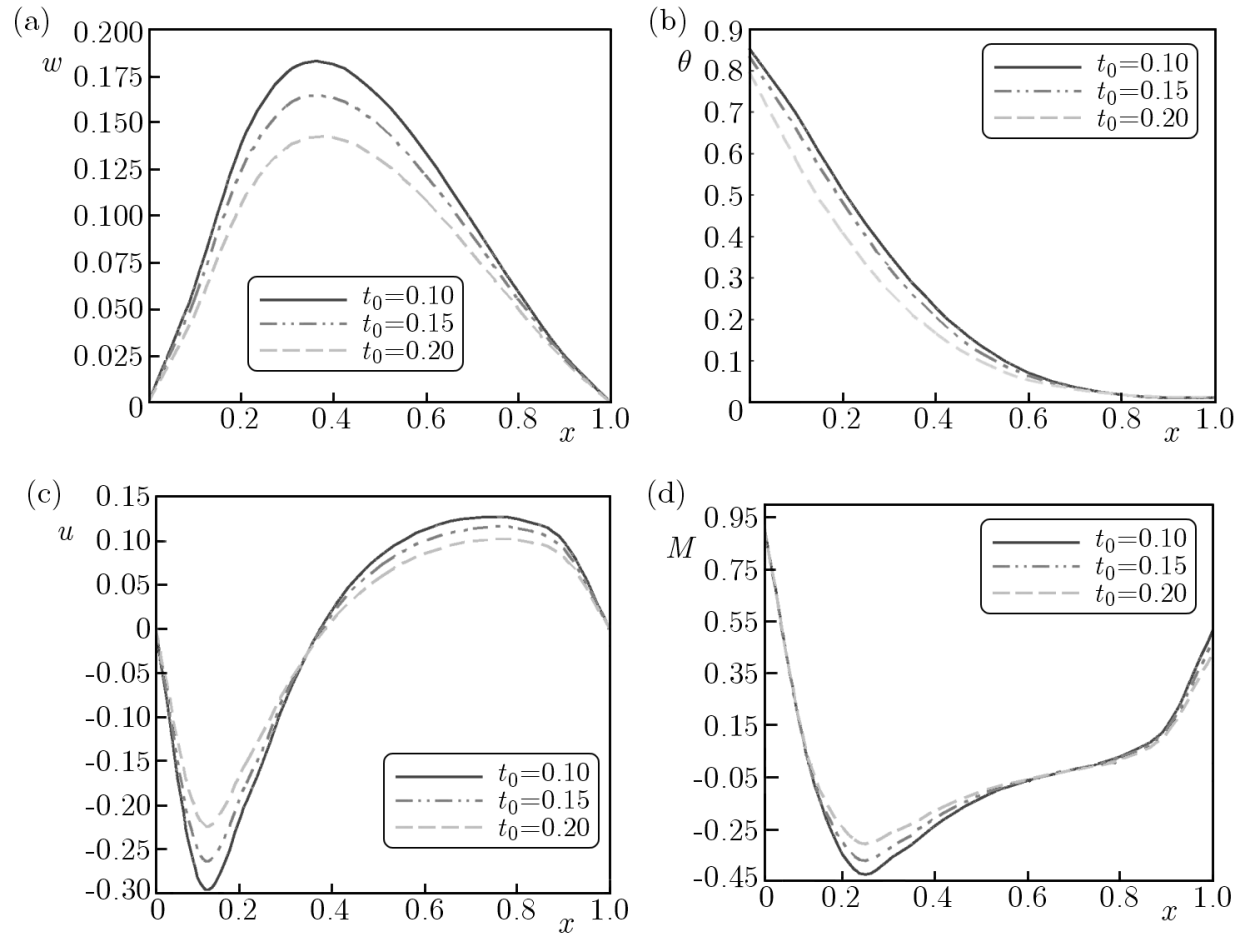

Fig. 3. The field quantities along the axial direction of the moving microbeam for different values of the pulse width $t_{0}$; (a) $w$ versus $x$, (b) $\theta$ versus $x$, (c) $u$ versus $x$, (d) $M$ versus $x$

In the last case, three different values of the dimensionless frequency of the external excitation $\omega$ are considered $\left(t_{0}=0.1, \bar{v}=1\right)$. The effects of $\omega$ on the vibration characteristics along the axial direction of the microbeam are shown in Fig. 4. The field quantities are very sensitive to the variation of the frequency of the external excitation $\omega$. The amplitudes of the wave of $w$ increase with an increase in $\omega$ and vice versa for the displacement $u$. The effect of the frequency of the external excitation $\omega$ on the bending moment $M$ is very small. The variation of $\omega$ has very low sensitivity to the temperature $\theta$.

\section{Conclusions}

In this work, the generalized thermoelastic problems of the axially moving microbeam subjected to sinusoidal pulse heating is studied. When very fast phenomena and small structure dimensions are involved, the classical law of Fourier becomes inaccurate. A more sophisticated model is then needed to describe mechanisms of thermal conduction in a physically acceptable way. Modern technology has enabled fabrication of materials and devices with characteristic dimensions of a few nanometers. The examples are super lattices, micro-wires, and quantum dots. At these length scales, the familiar continuum Fourier law for heat conduction is expected to fail due 


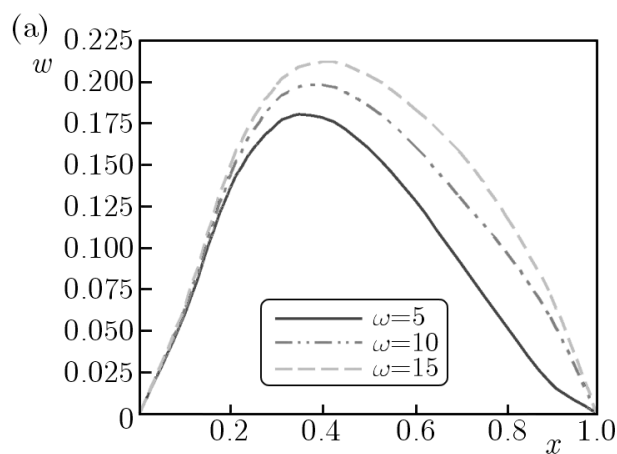

(b)
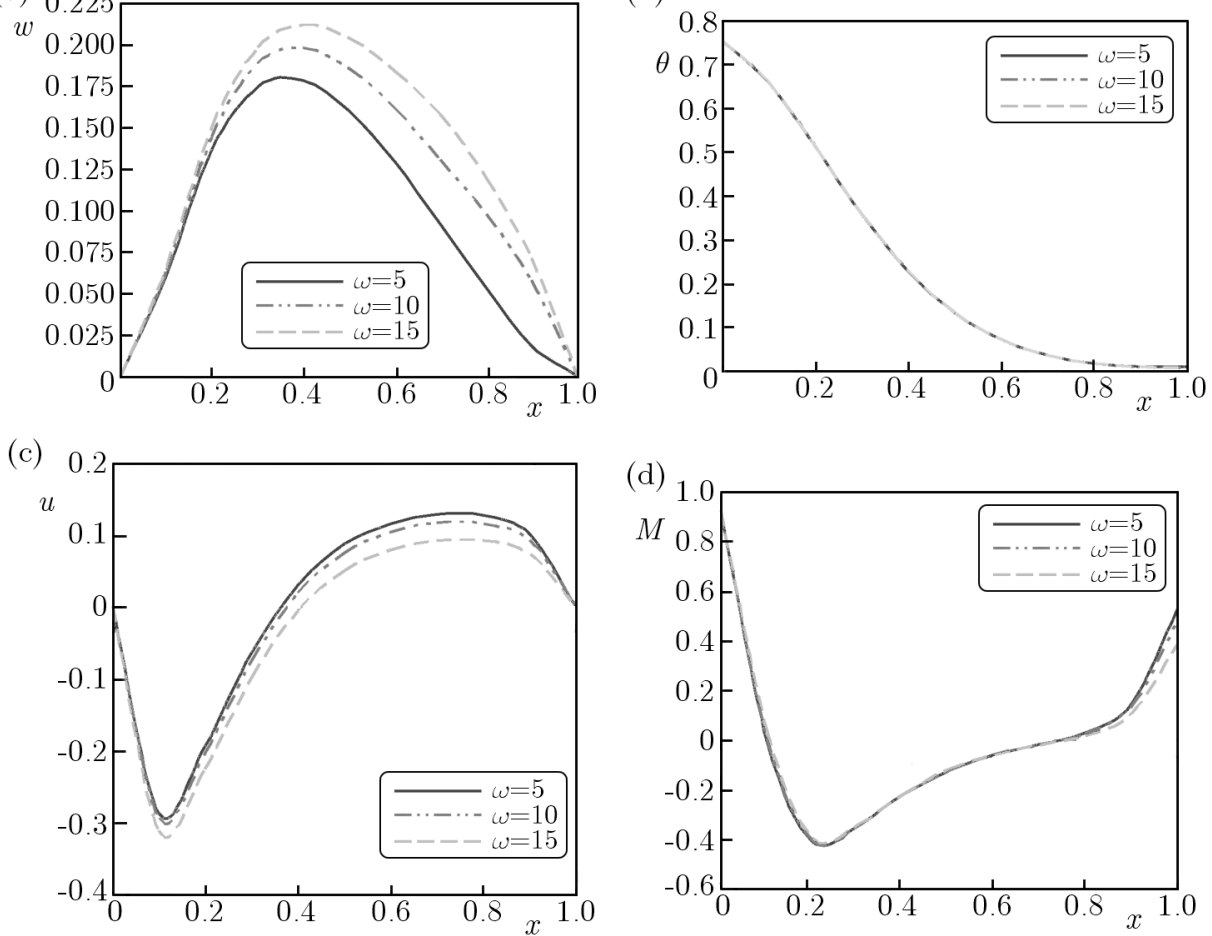

Fig. 4. Effect of the frequency of the external excitation $\omega$ on the field quantities along the axial direction of the moving microbeam; (a) $w$ versus $x$, (b) $\theta$ versus $x$, (c) $u$ versus $x$, (d) $M$ versus $x$

to both classical and quantum size effects. The generalized thermoelasticity theory with one relaxation time is used to solve this problem.

The general solutions are applied to a specific problem of an Euler-Bernoulli microbeam induced by sinusoidal pulse heating. An analytical technique based on the Laplace transform is used to calculate the vibration of deflection and the temperature. The effects of many parameters are studied and discussed. The effects of the moving transport speed $\bar{v}$ and frequency of the external excitation $\omega$ on the field variables are investigated. Moreover, the effects of the pulsewidth parameter $t_{0}$ of thermal vibration are examined.

We can formulate the following conclusions based on the above analysis:

- The waves reach the steady state depending on the speed of motion $v$.

- Note that $\bar{v}=0$ refers to an elastic Euler-Bernoulli beam.

- The pulse-width parameter has a significant effect on the field quantities.

- The frequency of the external excitation $\omega$ has significant effects on all the studied fields.

- The method used in the present article is applicable to a wide range of problems in thermodynamics and thermoelasticity.

- The results obtained in this paper may be considered as more general in the sense that they include the combined effect of graded and sinusoidal pulse heating.

- The theory of coupled thermoelasticity (CTE) can be extracted as a special case.

- The temperature converges to zero with increasing distance $x$, and all functions are continuous. The results show that the LS model of thermoelasticity predicts a finite speed of wave propagation that made the generalized theorem of thermoelasticity more consistent with the physical properties of the material.

- According to the results of this work, it is important to distinguish between dynamic deflections of the beam with and without axial movement. 
- Physical applications are found in the mechanical engineering, geophysical and industrial sectors.

- The results obtained in this paper may be considered as more general in the sense that they include the combined effect of moving sinusoidally varying heat pulse and frequency of the external excitation.

\section{References}

1. Avsec J., Oblak M., 2007, Thermal vibrational analysis for simply supported beam and clamped beam, Journal of Sound and Vibration, 308, 514-525

2. Biot M., 1956, Thermoelasticity and irreversible thermodynamics, Journal of Applied Physics, 27, 240-253

3. Chakraborty G., Mallik A.K., 2000, Wave propagation in and vibration of a traveling beam with and without non-linear effects, Journal of Sound and Vibration, 236, 277-290

4. Chang W.P., Wan S.M., 1986, Thermomechanically coupled nonlinear vibration of plates, International Journal of Non-Linear Mechanics, 21, 375-389

5. Chen L.Q., YAng X.D., 2005, Stability in parameteric resonace of axially moving viscoelastic beams with time-dependent speed, Journal of Sound and Vibration, 184, 879-891

6. Ding H., Chen L.-Q., 2011, Approximate and numerical analysis of nonlinear forced vibration of axially moving viscoelastic beams, Acta Mechanica Sinica, 27, 426-437

7. Green A.E., Lindsay K.A., 1972, Thermoelasticity, Journal of Elasticity, 2, 1-7

8. Houston B.H., Photiadis D.M., Vignola J.F., Marcus M.H., Liu X., Czaplewski D., Sekaric L., Butler J., Pehrsson P., Bucaro J.A., 2004, Loss due to transverse thermoelastic currents in microscale resonators, Materials Science and Engineering A, 370, 407-411

9. Kong L., Parker G., 2004, Approximate eigensolutions of axially moving beams with small flexural stiffness, Journal of Sound and Vibration, 276, 459-469

10. Kulkarni R.G., 2008, Solving sextic equations, Atlantic Electronic Journal of Mathematics, 3, $56-60$

11. Lord H.W., Shulman Y., 1967, A generalized dynamical theory of thermoelasticity, Journal of Mechanics and Physics of Solids, 15, 299-309

12. Manoach E., Ribeiro P., 2004, Coupled, thermoelastic, large amplitude vibrations of Timoshenko beams, International Journal of Mechanical Sciences, 46, 1589-1606

13. Mote C.M., JR., 1965, A study on band saw vibrations, Journal of The Franklin Institute, 279, $430-444$

14. Tabarrok B., Leech C.M., 1965, On the dynamics of an axially moving beam, Journal of The Franklin Institute, 279, 201-220

15. Tzou D.Y., 1996, Macro-to-Microscale Heat Transfer: the Lagging Behavior, Washington, DC, Taylor \& Francis

16. YANG X.D., 2007, Determination of the natural frequencies of axially moving beams by the method of multiple scales, Journal of Shanghai Jiaotong University, 11, 251-254

17. Zenkour A.M., Abouelregal A.E., 2014, The effect of two temperatures on a functionally graded nanobeam induced by a sinusoidal pulse heating, Structural Engineering and Mechanics, 51, 199-214

18. Zenkour A.M., Abouelregal A.E., Abbas I.A., 2014, Generalized thermoelstic vibration of an axially moving clamped microbeam subjected to ramp-type thermal loading, Journal of Thermal Stresses, 37, 1302-1323 